\title{
Planet formation around intermediate mass stars
}

\author{
Katherine A. Kretke ${ }^{1}$, D. N. C. Lin ${ }^{1,2}$ and Neal J. Turner ${ }^{3}$ \\ ${ }^{1}$ Department of Astronomy and Astrophysics, University of California, Santa Cruz, CA \\ ${ }^{2}$ Kavli Institute of Astronomy and Astrophysics, Peking University, Beijing, China \\ ${ }^{3}$ Jet Propulsion Lab, Pasadena, CA
}

\begin{abstract}
We present a mechanism by which gas giants form efficiently around intermediate mass stars. MRI-driven turbulence effectively drives angular momentum transport in regions of the disk with sufficiently high ionization fraction. In the inner regions of the disk, where the midplane temperature is above $\sim 1000 \mathrm{~K}$, thermal ionization effectively couples the disk to the magnetic field, providing a relatively large viscosity. A pressure maximum will develop outside of this region as the gaseous disk approaches a steady-state surface density profile, trapping migrating solid material. This rocky material will coagulate into planetesimals which grow rapidly until they reach isolation mass. Around intermediate mass stars, viscous heating will push the critical radius for thermal ionization of the midplane out to around $1 \mathrm{AU}$. This will increase the isolation mass for solid cores. Planets formed here may migrate inwards due to type II migration, but they will induce the formation of subsequent giant planets at the outer edge of the gap they have opened. In this manner, gas giants can form around intermediate mass stars at a few AU.
\end{abstract}

Keywords. planetary systems: formation, protoplanetary disks

\section{Introduction}

Most surveys to detect extrasolar planets using radial velocity (RV) techniques have focused on solar type stars due to their favorable spectral characteristics. While on the main sequence, intermediate mass stars ( stars with $1.5 M_{\odot} \lesssim M_{*} \lesssim 3 M_{\odot}$ ) make poor RV candidates as they only have a small number of spectral lines, and those tend to be relatively broad. However, once these stars evolve off the main sequence their cooler, slower rotating outer layers make them more suitable targets. Recent RV surveys targeting evolved intermediate mass stars suggest that planets around these stars have interesting distinctions from those around solar type stars (Lovis \& Mayor 2007). The frequency of giant planets may be higher around intermediate mass stars. Also, they apparently lack closer in planets, even when taking into account planets whose orbits have been disrupted by stellar evolution. (Johnson 2007).

In order to understand these differences we look at the structure of the protoplanetary disk from which the planets must have formed. In the core accretion gas-capture model of planet formation (eg. Bodenheimer \& Pollack 1986) gas giant formation requires a large $\sim 10 M_{\oplus}$ solid core to form in the presence of the gaseous disk. Therefore the we must understand how solids migrate and are retained in gaseous disks in order to understand the distribution of gas giant planets.

In most regions of the disk, the midplane gas pressure, $P_{\text {mid }}$, decreases with $r$ so that the azimuthal velocity of the gas, $V_{\phi}$, is sub-Keplerian. Grains larger than a few $\mathrm{cm}$ are decoupled from the gas and move at Keplerian speeds so they experience head winds and undergo orbital decay (Weidenschilling 1977). However if there are irregularities in the disk then the particles will instead drift towards local pressure maxima (Haghighipour \& Boss 2003).

The inner regions of typical protostellar disks are thermally ionized (Umebayashi 1983). This ionization couples the disk gas to any existing magnetic field and, in a differentially rotating 


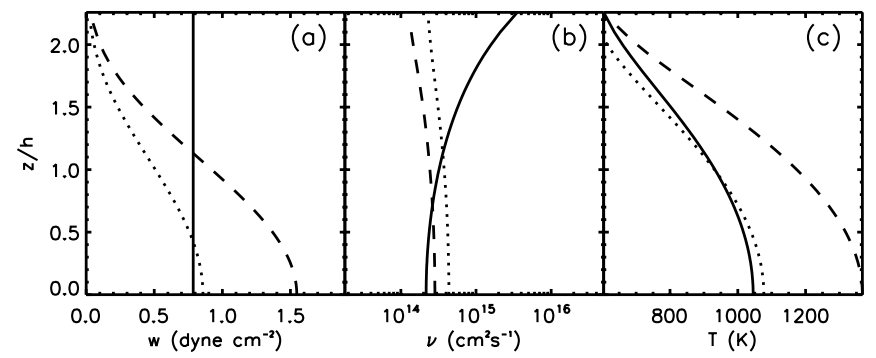

Figure 1. A comparison of this new more realistic parametrization of MRI vertical viscosity (solid line) to a disk with constant viscosity as a function of height with two different " $\alpha$ s (dashed and dotted lines). See text for details. The panel (a) shows the accretion stress, panel (b) the viscosity, and panel (c) the temperature

disk, the magneto-rotational instability (MRI; Balbus \& Hawley 1991) generates turbulence. This turbulence provides a source of viscosity which transports angular momentum in the disk. At greater distances from the star, thermal ionization is insufficient for MRI turbulence to develop. Here stellar x-rays and diffuse cosmic rays may ionize the surface layers of the disk, resulting in a viscously active turbulent surface sandwiching an inactive "dead zone" (Gammie 1996).

In section 2 we discuss the vertical structure of an MRI active disk using a more realistic approximation of the disk viscosity as a function of height and use this parametrization to calculate the location of the inner edge of the deadzone as a function of stellar mass and mass accretion rate. In section 3 we estimate the amount of solid material which can accumulate in this region and discuss the implications for the formation of gas giant cores.

\section{Thermal Structure of MRI Active Disks}

The standard Shakura \& Syunyaev 1973 parametrization of the viscosity in geometrically thin accretion disks $\left(\nu=\alpha c_{s}^{2} \Omega_{K}\right)$ is sometimes extended into two dimensions by using the local temperature and maintaining a constant $\alpha$ with height, which leads to a constant viscosity with height in an isothermal disk (sometimes known as the " $\alpha P$-formalism"; e.g. Cannizzo 1992). However, MHD simulations demonstrate that in MRI active regions the viscosity is not constant with height. A better approximation is that the accretion stress $w=(3 / 2) \nu \rho \Omega_{K}$ is constant with height for 2 disk scale heights, above which $w$ decreases significantly (Miller \& Stone 2000). Therefore we parametrize the viscosity in an $\alpha$-type manner as

$$
\nu(z)=\frac{2}{3} \frac{\alpha P_{\mathrm{mid}}}{\rho(z) \Omega_{K}} .
$$

using the midplane density instead of the more common $2 \mathrm{D}$ parametrization using the local pressure and density. Figure 1 compares the usual assumption that viscosity is proportional to the local sound-speed with the assumption that the accretion stress is constant. The solid curves show the disk structure in the case of a constant $w$, while the dashed and dotted curves are for $v \propto c_{s, l o c a l}$, but for two different values for the constant of proportionality $\alpha$. The dashed curve shows the structure for a disk with the same $\alpha$ as the constant $w$ case, such that if the two disks had the same midplane temperature then the viscosity at the midplane would be the same. The dotted curve is scaled such that the mass accretion rate through the annulus is the same as the constant $w$ case.

In a disk with a steady-state mass accretion rate, the mass accretion rate through each radius is

$$
\dot{M}=2 \pi \int_{-\infty}^{\infty} \rho \nu d z
$$

If one uses the same $\alpha$ parameter in the constant $w$ and standard local description of viscosity 
(Figure 1 dashed line), then the midplane temperature in the later case is significantly warmer than in the more realistic approximation, in agreement with Hirose et al. 2006. However, we can define a $\alpha_{\text {eff }}$ based on the midplane temperature similar to the original Shakura \& Syunyaev model, then this can be related to the mass accretion rate as given by Pringle 1981

$$
\alpha_{\mathrm{eff}}=\frac{\dot{M} \Omega_{K}}{3 \pi c_{s, \text { mid }}^{2} \Sigma} .
$$

Adjusting $\alpha$ so that the two different parameterizations of viscosity have the same $\alpha_{\text {eff }}$ yields a cooler midplane as the surface density is smaller for the same mass accretion rate (Figure 1 dotted line). The temperature differences are not as dramatic in this case, but the disk is still less isothermal than the constant $w$ situation.

\subsection{Vertical Structure}

We solve the disk structure of an optically thick, viscously heated disk with radiative energy transport. Along with equations for continuity and hydrostatic equilibrium the disk structure is describe by the following equations:

$$
\begin{aligned}
\frac{d F_{E}}{d \sigma} & =\frac{9}{8} \nu \Omega_{K}^{2} \\
\frac{d T}{d \sigma} & =-\frac{F_{E}}{2 \rho k} \\
\frac{d z}{d \sigma} & =\frac{1}{2 \rho} \\
\frac{d P}{d \sigma} & =-\frac{1}{2} \Omega_{K}^{2} z
\end{aligned}
$$

where $F_{E}$ is the radiative flux, $\sigma(z)$ is the column depth of material between height $z$ and $-z$, and

$$
k=k_{\mathrm{rad}}=\frac{4 a c}{3} \frac{T^{3}}{\kappa \rho} .
$$

In the inner regions of a rapidly accreting disk the surface density and optical depth of the disk is large enough that solving the disk structure below the photosphere $\left(z_{e}\right)$ is sufficient for studying most of the disk mass. We can then use photospheric boundary conditions $T\left(z_{e}\right)=$ $T_{i r r}+\left(F_{E} / \sigma_{s b}\right)^{4}$ and $P\left(z_{e}\right)=(2 / 3) \Omega_{K} z_{e} / \kappa$. We use a constant opacity $\kappa=1 \mathrm{~cm}^{2} \mathrm{~g}^{-1}$ consistent with opacities from Ferguson et al. 2005.

For high mass accretion rates, the midplane temperatures at this region is dominated by viscous heating. Therefore we use a simple prescription for stellar irradiation, simply assuming a flat disk (see Chiang \& Goldreich 1997)

$$
T_{e}=\left(\frac{2}{3 \pi}\right)^{1 / 4}\left(\frac{R_{*}}{a}\right)^{3 / 4} T_{*} .
$$

For the stellar parameters we use $1 \mathrm{Myr}$ old stars with solar metallicity from D'Antona \& Mazzitelli 1997. The 2D structure of the disk may have interesting effects on the amount of stellar light intercepted, for example a "wall" of material at the inner edge of the deadzone. But as the $1+1 \mathrm{D}$ approximation cannot explicitly address this issue we leave this problem for future studies.

\subsection{Location of the inner edge of the Deadzone}

Solving the equations for vertical structure we can calculate the location at which the midplane just satisfies the ionization criteria of $x_{e} \geqslant 10^{-12}$ (which corresponds to $T \sim 1000 \mathrm{~K}$ ) (see Klahr et al. 2006) for different surface densities. We calculate the mass accretion rate through that annulus using equation 2.2 with $\nu(z)$ from equation 2.1. In Figure 2 the curves show the location of the inner edge of the deadzone as a function of stellar mass and accretion rate assuming that 


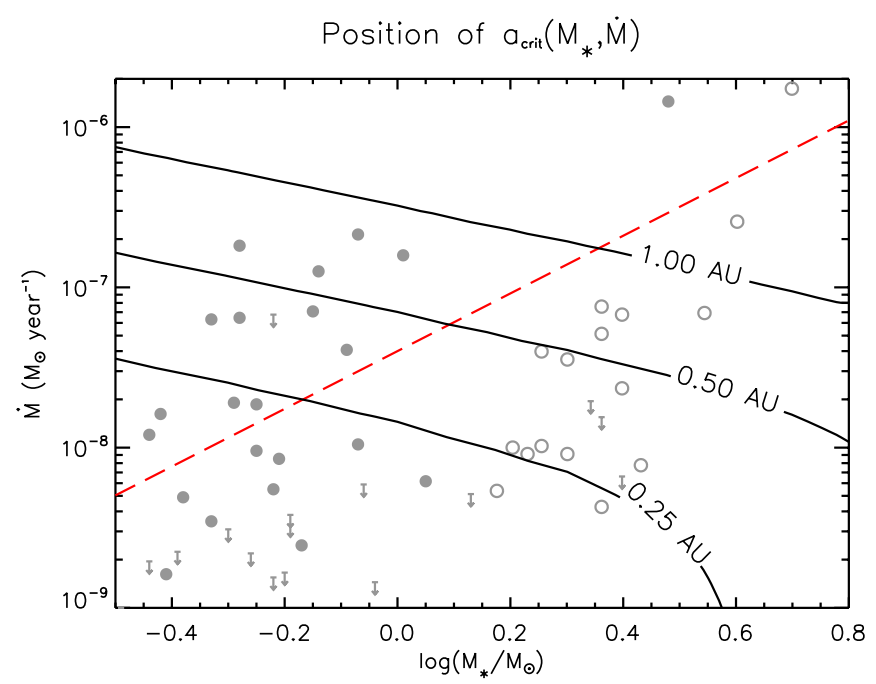

Figure 2. The curves show the position of the inner edge of the deadzone as a function of stellar mass and mass accretion rates. Symbols represent observations of mass accretion rates for stars of various masses (see text for details). The dashed line shows the best-fit for the $\rho$-Oph cluster from Natta et al. 2006.

in the active region $\alpha=10^{-2}$. For most values of $M_{*}$ and $\dot{M}$ these theoretical curves can be approximated by

$$
a_{\text {crit }}=0.22 A U\left(\frac{\dot{M}}{10^{-8} M_{\odot} \text { year }^{-1}}\right)^{0.42}\left(\frac{M_{*}}{M_{\odot}}\right)^{0.32} .
$$

The empirical relation between stellar mass and mass accretion rate is still rather uncertain (and shows significant scatter), so for reference the symbols in Figure 2 indicate measurements of mass accretion rates onto young stars. The solid points are from Natta et al. 2006 observations of $\rho$-Oph and the dashed line shows the best fit to their data which is $\dot{M} \propto M_{*}^{1.8}$. As the mass accretion rate for the higher mass stars is dominated by a single object, we have also plotted as open points from the older, heterogeneously distributed intermediate mass stars (Garcia Lopez et al. 2006). The estimated ages on these stars range from 1-10 Myr, so they are systematically older than $\rho$-Oph, and when they were younger they may have had higher mass accretion rates consistent with the extrapolation from $\rho$-Oph (Garcia Lopez et al. 2006). Using the best fit relationship from $\rho$-Oph implies that $a_{\text {crit }} \propto M_{*}^{1.1}$. Clarke \& Pringle 2006 suggest that the correlation between stellar mass and mass accretion rate may not be this steep due to observational biases. If instead we use their estimation that $\dot{M} \propto M_{*}, a_{\text {crit }} \propto M_{*}^{0.74}$ becomes slightly less sensitive to $M_{*}$.

\section{Planet Formation at the Inner Edge of the Deadzone}

Few mechanisms for angular momentum transport in protoplanetary disks are as efficient as MRI, therefore we expect the disk viscosity to decrease in the deadzone, which will cause a corresponding increase in the surface density of the disk if the disk can reach a steady-state surface density profile (Kretke \& Lin 2007). This change in the surface density will create a local pressure maximum capable of accumulating solids. The $a_{\text {crit }}$ calculated in the previous section corresponds to the outermost thermally ionized location. The location of the pressure maxima will be larger than $a_{\text {crit }}$ by at least one scale height and perhaps more depending on how the details of the $2 \mathrm{D}$ structure which is neglected here. 

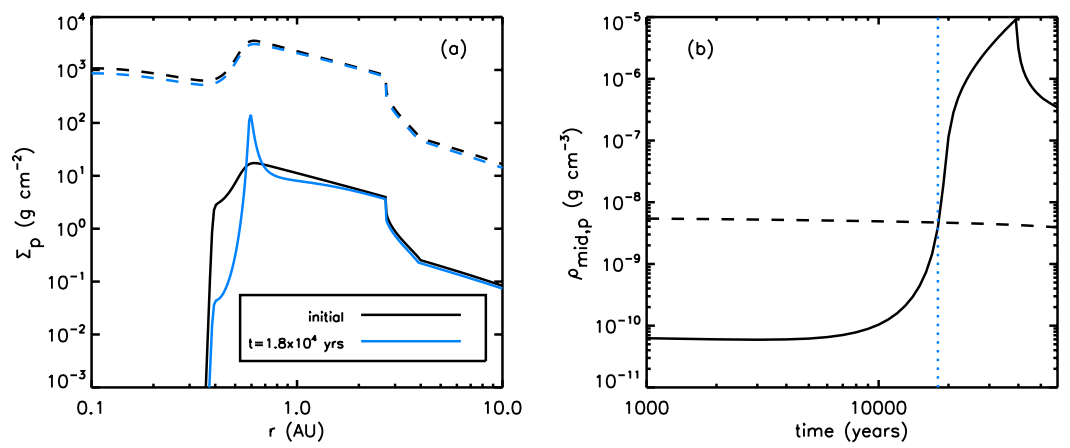

Figure 3. Panel (a) shows the of the solid surface density (solid curve) and the gas surface density (dashed curve) at two different times. Panel (b) shows the evolution of the midplane density of the two fluids at the pressure maximum. The vertical dotted line marks the time when the midplane gas and solid densities are equal.

\subsection{Accumulation of Solids}

In order to estimate the amount of material which will accumulate at the inner edge of the deadzone we use Garaud 2007 model (modified to have an $\alpha_{\text {eff }}$ which varies with radius) to estimate the migration of material in a gaseous disk. This model approximates the evolution of solids in a disk by following the growth and migration of solids through an evolving gaseous disk. Particles undergo radial migration due to gas drag, and the radial velocity of the solids with respect to the gas is given by

$$
v_{p}=-\frac{h^{2}}{a^{2}} v_{K} \frac{\partial \ln (P)}{\partial \ln (a)} \frac{2 \pi S t(s)}{\left[4 \pi^{2} S t^{2}(s)+1\right]}
$$

where $v_{K}$ is the Keplerian velocity and the Stokes number $S t(s)$ is the ratio of the local stopping time to the local orbital time for particles of radius $s$ (See Garaud 2007 for more details). For illustrative purposes, Figure 3 demonstrates the buildup of material in less than $10^{5}$ years from an initially well-mixed disk of micron sized grains. The initial gas profile is for a steady-state mass accretion rate of $\dot{M}=4 \times 10^{-8} M_{\odot}$ year $^{-1}$ with $\alpha_{\mathrm{eff}}=10^{-2}$ in the active regions and $\alpha_{\text {eff }}=10^{-3}$ in the MRI dead regions. At the pressure maximum at inner edge of the dead zone material accumulates rapidly once the particles grow to meter-sized, mobile objects. Particles will continue to accumulate until the midplane particle density $\left(\rho_{\mathrm{mid}, p}\right)$ becomes comparable to the midplane gas surface density at the inner edge of the deadzone. At this point the solid material will force a larger region of the gas to rotate at Keplerian velocity increasing the radial extent of the gas moving at Keplerian speed and spreading out the location where particles accumulate. This makes the accumulation of particles a self-limiting process, but only once the solid surface density increases by an order of magnitude. This feedback of the particles on the gas is neglected in this contribution but will be included in future work. For these calculations we simply assume that the order-of-magnitude increase of solid surface density which should accumulate without problem is a reasonable approximation for the amount of solid material present at the inner edge of the deadzone.

\subsection{Core Formation}

In order to estimate the size of cores formed at the inner edge of the deadzone we use the expression from Ida \& Lin 2005 for core isolation mass.

$$
M_{c, i s o} \simeq 0.16\left(\frac{\Sigma_{d}}{10 \mathrm{~g} \mathrm{~cm}^{-2}}\right)^{3 / 2}\left(\frac{a}{1 \mathrm{AU}}\right)^{3}\left(\frac{M_{*}}{M_{\odot}}\right)^{-0.5} M_{\oplus}
$$

This expression assumes that the planetesimal surface density $\left(\Sigma_{d}\right)$ is uniform enough throughout the embryo's feeding zone to be approximated by a single value. By assuming the solids scale 


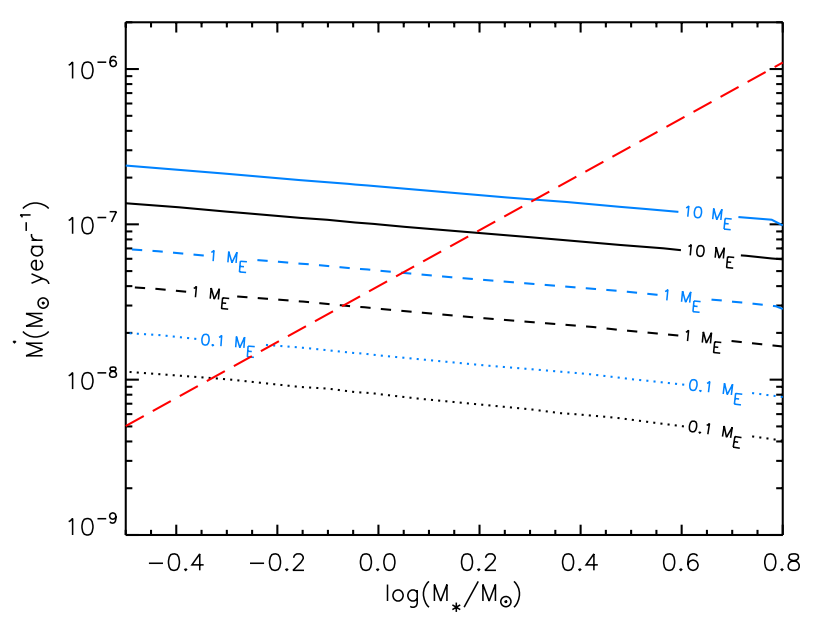

Figure 4. The core isolation mass at the inner edge of the deadzone assuming $\alpha_{\text {eff }}=10^{-4}$ (in the deadzone) and $f=0.05$ (black curves). The blue curves show how the core isolation mass would change if either $f$ is decreased or $\alpha_{\text {eff }}$ increased by a factor of two. The long-dashed line is the same as in Figure 2 for reference.

with a steady-state gas surface density in the deadzone

$$
\Sigma_{d}=f \Sigma_{g}=f \frac{\dot{M} \Omega_{K}}{3 \pi \alpha_{\mathrm{eff}} c_{s}^{2}} .
$$

Here $\alpha_{\text {eff }}$ includes a large contribution from the deadzone, so it will be significantly smaller than in the thermally ionized regions. If a sufficient column of the surface layers of the disk is active to sustain MRI turbulence then the deadzone will also sustain a small degree of accretion stress even though it is laminar (Fleming \& Stone 2003). We note that the total surface density at this region is sensitive to the viscous parameters, therefore the normalization may be scaled, but the trend in stellar mass should remain.

The time to reach this isolation mass is also given by Ida \& Lin 2005 and is approximately

$$
\begin{aligned}
t_{\text {core }}=0.26\left(\frac{\Sigma_{d}}{10 \mathrm{~g} \mathrm{~cm}^{-2}}\right)^{-1 / 2} & \left(\frac{\Sigma_{g}}{3.4 \times 10^{3} \mathrm{~g} \mathrm{~cm}^{-2}}\right)^{-1 / 2} \\
& \left(\frac{a}{1 \mathrm{AU}}\right)^{8 / 5}\left(\frac{M_{*}}{M_{\odot}}\right)^{1 / 3} \mathrm{Myr} .
\end{aligned}
$$

This time is typically less than the viscous evolution time of the disk so cores should reach isolation mass. By assuming that $\alpha_{\mathrm{eff}}$ is independent of stellar mass, the viscosity is the same at $a_{\text {crit }}$ for all stellar masses. This implies that a higher $\dot{M}$ is due to a higher surface density. The fact that typical disk mass accretion rates are higher for more massive stars and that the location of the pressure inversion is farther out combine to make the core size rise steeply with stellar mass. The isolation mass of the core at $a_{\text {crit }}$ goes as $M_{c, i s o} \propto \dot{M}^{1.8} M_{*}^{0.5}$. Using the best fit relationship for the relationship between stellar mass and mass accretion rate derived from $\rho$-Oph fit, $M_{c, i s o} \propto M_{*}^{3.7}$.

Using Lodders 2003 solar composition, most of the silicates and metals will still be in solid phase at $1000 \mathrm{~K}$. Therefore the mass of solids should be a little less than the $0.5 \%$ of gas at this region. This implies that the base dust-to-gas ratio would be $f=0.005$. However, if the solid material accumulates in accordance with the results in Figure 3, $f$ will increase by an order of magnitude. Figure 4 shows the core isolation mass for $f=0.05$ and $\alpha_{\mathrm{eff}}=10^{-4}$, for comparison, the blue lines show the isolation mass for the equivalent situation of $f$ decreased or $\alpha_{\text {eff }}$ begin increased by a factor of two. 


\subsection{Gas Giant Formation}

With this estimate of core isolation mass we can continue using the approximations of Ida \& Lin 2005 to look at further potential growth. Gas capture becomes important once the planet has reached core isolation mass. The planet growth due to gas capture is

$$
\frac{d M_{p}}{d t} \approx \frac{M_{p}}{\tau}
$$

and

$$
\tau \approx \tau_{0}\left(\frac{M_{p}}{M_{\oplus}}\right)^{-3}
$$

where $\tau_{0} \approx 10^{10}$ years. As long at the final mass of the planet is much greater than the original core mass, the timescale for planet formation is

$$
t_{\text {giant }} \approx \frac{\tau_{0}}{3}\left(\frac{M_{c}}{M_{\oplus}}\right)^{-3} .
$$

This effectively means that in order for a gas giant to form within the lifetime of the evolving disk, the core isolation mass must be on the order of $10 M_{\oplus}$. Using the relationship derived for the core mass this implies that $t_{\text {giant }} \propto \dot{M}^{-5.4} M_{*}^{-1.5}$. This implies $t_{\text {giant }} \propto M_{*}^{-11}$ using the $\rho$-Oph data. This very steep function demonstrates that giant planets will not form effectively at the inner edge of the deadzone around less massive stars, but may form around higher mass stars with higher accretion rates.

\section{Summary and Discussion}

Observations indicate that planets may form systematically more efficiently around intermediate mass stars and their semi-major axis appear to be systematically larger than those around solar type stars to a degree which may not be completely explained by post-main sequence evolution. In this paper we demonstrate a mechanism to form planets preferentially around intermediate mass stars. Solid material can collect at the pressure inversion at the inner edge of the deadzone in protoplanetary disks. In order for the material accumulated to form gas giant cores, the inner edge of the deadzone must be sufficiently far from the host stars. This condition is only likely to be met around intermediate mass stars with high accretion rates.

This model does assume that the location of the inner edge of the deadzone is relatively constant with time, and least until the solids grow in size large enough to be effectively decoupled from the gas. Wünsch et al. 2006 use different parameterizations of vertical viscosity and find that the inner edge of the deadzone may be unstable to oscillations under some conditions. This may help explain why excess solids are not found at the inner edge of the deadzone in all disks. Once the first planet has formed and grown large enough to open a gap, then the outer edge of the gap provides another pressure maximum which may trap solids and encourage the growth of the next planet (Bryden et al. 2000). Therefore some of the observed planets may be subsequent planets formed in the systems, so they may be located farther out than the location of the original pressure maximum at the inner edge of the deadzone.

\section{References}

Balbus, S. A. \& Hawley, J. F. 1991, ApJ, 376, 214

Bodenheimer, P. \& Pollack, J. B. 1986, Icarus, 67, 391

Bryden, G., Różyczka, M., Lin, D. N. C., \& Bodenheimer, P. 2000, ApJ, 540, 1091

Cannizzo, J. K. 1992, ApJ, 385, 94

Chiang, E. I. \& Goldreich, P. 1997, ApJ, 490, 368

Clarke, C. J. \& Pringle, J. E. 2006, MNRAS, 370, L10

D’Antona, F. \& Mazzitelli, I. 1997, Memorie della Societa Astronomica Italiana, 68, 807

Ferguson, J. W., Alexander, D. R., Allard, F., Barman, T., Bodnarik, J. G., Hauschildt, P. H., Heffner-Wong, A., \& Tamanai, A. 2005, ApJ, 623, 585 
Fleming, T. \& Stone, J. M. 2003, ApJ, 585, 908

Gammie, C. F. 1996, ApJ, 457, 355

Garaud, P. 2007, ArXiv e-prints, 705

Garcia Lopez, R., Natta, A., Testi, L., \& Habart, E. 2006, A\&A, 459, 837

Haghighipour, N. \& Boss, A. P. 2003, ApJ, 598, 1301

Hirose, S., Krolik, J. H., \& Stone, J. M. 2006, ApJ, 640, 901

Ida, S. \& Lin, D. N. C. 2005, ApJ, 626, 1045

Johnson, J. A. 2007, ArXiv e-prints, 710

Klahr, H., Różyczka, M., Dziourkevitch, N., Wünsch, R., \& Johansen, A. 2006, Turbulence in protoplanetary accretion disks: driving mechanisms and role in planet formation. (Planet Formation), 42

Kretke, K. A. \& Lin, D. N. C. 2007, ApJL, 664, L55

Lodders, K. 2003, ApJ, 591, 1220

Lovis, C. \& Mayor, M. 2007, A\&A, 472, 657

Miller, K. A. \& Stone, J. M. 2000, ApJ, 534, 398

Natta, A., Testi, L., \& Randich, S. 2006, A\&A, 452, 245

Pringle, J. E. 1981, ARA\&A, 19, 137

Shakura, N. I. \& Syunyaev, R. A. 1973, A\&A, 24, 337

Umebayashi, T. 1983, Progress of Theoretical Physics, 69, 480

Weidenschilling, S. J. 1977, MNRAS, 180, 57

Wünsch, R., Gawryszczak, A., Klahr, H., \& Różyczka, M. 2006, MNRAS, 367, 773 